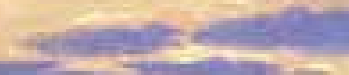

\section{Neues zu Caspar David Friedrichs "Elblandschaft"}

\author{
Herrmann Zschoche
}

Das Bildchen „Elblandschaft“ im Kulturhistorischen Museum Stralsund gehört zu einer Reihe kleinformatiger Werke, die in den 1820er und 1830er Jahren häufiger von Caspar David Friedrich gemalt worden sind.

Anscheinend war es nie ausgestellt, und über die Provenienz wissen wir wenig. Wahrscheinlich wurde es 1862 mit der Sammlung Dr. Hillig, Leipzig, versteigert und war dort beschrieben als „Morgenlandschaft, auf einem quer durch das Bild laufenden Dammweg gehen zwei Bäuerinnen“. Bevor es ins Museum gelangte, war es im Besitz der Witwe des Greifswalder Altermanns Odebrecht. ${ }^{1}$ Basis des Bildes ist eine Skizze vom September $1824^{2}$, deren Vordergrund im Gemälde zu steinigem Erdreich und einem Hügel mit blühenden Pflanzen ausgearbeitet ist. Dahinter eine in der Skizze nicht definierte helle Fläche, die man im Gemälde als Flüsschen deuten könnte, das von einem Damm begrenzt wird. Die beiden Frauen sind auf der Skizze noch kaum als solche zu erkennen. Im Gemälde gehen sie nebeneinander in der Bildmitte. Die eine, in rotem Rock mit rotem Kopftuch, trägt einen Wäschekorb, die andere, in blauem Kleid mit weißem Kopftuch, geht nun gebeugt unter der Last des Reisigbündels. Auch die im Gemälde weggelassene Architektur am rechten Rand der Skizze gibt keinen Hinweis auf die dargestellte Örtlichkeit, die wohl in der weiteren Umgebung Dresdens zu suchen ist. Für den Hintergrund wurde eine 1806 entstandene Zeichnung verwendet, die den Blick zum bereits jenseits der sächsischen Grenze in Böhmen liegenden Hohen Schneeberg zeigt. Friedrichs Hauptinteresse beim Skizzieren galt
Caspar David Friedrich: Elblandschaft. Um 1825.

Öl auf Leinwand, 22,2 x 30,5 cm 


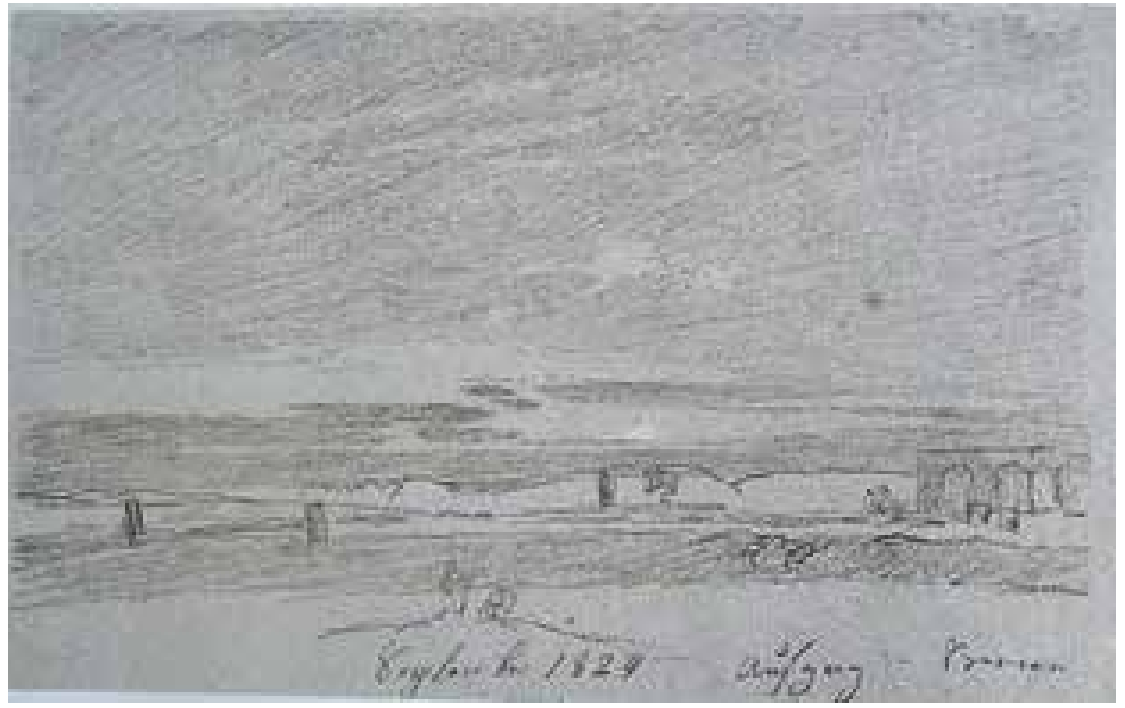

Caspar David Friedrich: Sonnenaufgang, September 1824. Bleistift, weiße Kreide, $12,4 \times 20,2 \mathrm{~cm}$. (Privatbesitz)

2 Christina Grummt: Caspar David Friedrich. Die Zeichnungen. München 2011, Band II, Kat.Nr. 1009 .

3 Börsch-Supan (wie Anm. 1)

Zschoche 2006, Brief 106

5 Börsch-Supan (wie Anm. 1), Kat.-Nr. 273

6 Grummt (wie Anm. 2), Kat.-Nr. 899. offenbar dem hohen Himmel, der durch Weißhöhungen schon malerisch angelegt ist. Das 2011 erstmals veröffentlichte Blatt ist von Friedrich mit „Aufgang der Sonne“ bezeichnet. Dem entspricht die offenbar aus der Erinnerung wiedergegebene kühle Farbigkeit der Malerei, in der blaue und zart violette Töne vorherrschen.

Noch in Unkenntnis der Skizze, wurden die beiden älteren Frauen auf dem Dammweg von Helmut Börsch-Supan christlich-symbolisch als am baldigen Ende ihres Lebensweges gesehen. „Es dürfte eine Abendlandschaft gemeint sein, da das Motiv der Reisig schleppenden Bäuerin [...] bei einer Morgenlandschaft wenig sinnvoll ist. Der Dammweg, der den irdischen Vordergrund scharf vom jenseitigen Hintergrund trennt, symbolisiert den Lebensweg des Menschen als eines beiden Bereichen zugehörigen Wesens. Die Bäuerinnen gehen, unter der Last ihres Schicksals gebeugt, dem Tod entgegen.“3 $\mathrm{Zu}$ mindest von der Tageszeit her ist diese Lesart nun nicht mehr zu stützen.

Sinnverwandt erscheint der „Herbstabend mit Reisigsammler“ 1826 wird das Bildchen von einem Schüler Friedrichs beschrieben: „Im Hintergrund, in dichtem Nebel verhüllt, ein Berg, davor Wald und ein Dorf, im Vorgrund weiter nichts als gepflügtes Land. Auf einem engen Rasenstreifen geht ein Mann, der mühsam Dornen nachschleppt, vor ihm fliegen Raben in die Höhe und verlieren sich im Hintergrund. Nun hättest Du hören sollen, was die Menschen für Allegorien auf das menschliche Leben und dessen Mühseligkeiten machten, wie tief erdacht das alles sei. Ich hasste alles, was darüber gesagt wurde. Ohne weiter an alle Allegorien zu denken, zog mich der so unglaublich treu und wahr dargestellte Herbstabend so an, dass ich wohl eine halbe Stunde davor gestanden habe, ohne mich davon trennen zu können. Ich fragte ihn, wie er dazu gekommen sei, dass sein Bild, wo doch so wenig da wäre, so fesseln könne. ,Eben so, wie die Natur Sie fesselt', sagte er, ,denn das Bild ist bis auf den kleinsten Grashalm treu nach der Natur gemalt, alles so, wie Sie es da sehen.' Er geht den Abend spazieren, sieht hinter dem Großen Garten bei Dresden dies alles und malt es am nächsten Tag. Und auf diesem Wege entstehen seine meisten Bilder." 4

Indes wissen wir, dass die meisten Bilder Friedrichs aus zu verschiedener Zeit an verschiedenen Orten gewonnenen Natureindrücken zusammengesetzt sind. Zweifellos leiteten ihn dabei bestimmte Vorstellungen und Absichten. Hier ist es der in die realistische Szenenerie dominant eingefügte Reisigsammler, in dem von anderen mit Recht eine Allegorie auf das menschliche Leben und dessen Mühseligkeiten gesehen wurde. Es ist evident, dass Friedrich seine Kunst von einem religiösen Standpunkt sah. Es scheint aber ein „Bedeutungsgefälle“ zu geben, und nicht jedes Bild lässt sich einordnen in ein vermutetes System durchgängiger religiöser Symbolik.

Das Gegenstück der „Elblandschaft“ wird 1862 als Abendbild beschrieben: „Kiefernwald bei aufgehendem Mond, im Vordergrund ein schilfbewachsener Teich mit einem Fischer; dahinter am Waldrand eine Hütte, vor der ein Feuerchen brennt." ${ }^{5}$

Auf beiden Bildern sind arbeitende Menschen dargestellt, die Frauen am Morgen ihres Arbeitstages, der Fischer am Abend.

Der sichere Ertrag bei näherer Betrachtung der Elblandschaft ist folgender: sie hat mit der Elbe nichts zu tun, und entstand frühestens im September 1824. Dieses Datum trägt auch die Studie eines Mannes mit Hund, mit Notizen über die Farben des Himmels, „des Abends gezeichnet, gegen Morgen [d. h. gegen Osten] gewendet" 6 Auch die wenigen Ölskizzen Friedrichs zeigen die Lichtphänomene abendlicher Himmel, und entstanden 1824, wohl unter dem Einfluss Johan Dahls. Auch die um diese Zeit enge Beziehung zu Carl Gustav Carus, mit dem er in der Umgebung Dresdens zeichnend unterwegs war, könnte eine Rolle gespielt haben.

\section{Morgen oder Abend?}

Die Beantwortung der Frage gilt in der FriedrichForschung als wichtiger Anhaltspunkt für die Interpretation der Bilder. Altphilologe Karl Morgenstern aus Dorpat besucht im Herbst 1808 Friedrichs Atelier, und notiert zu dem noch nicht ganz vollendeten „Kreuz im Gebirge“ (Tetschener Altar): „... ein Crucifix. Dahinter die aufsteigende Sonne, in welcher es gesehen wird“. Basilius von Ramdohr schreibt zum selben Bild: „Ein anderer Fehler ist der, daß die Tageszeit zweifelhaft bleibt, vermöge des Abgangs [Fehlens] aller Flächen. Für den Morgen spricht die Kälte der Luft, gegen ihn der Mangel an Nebel. Diesen Ab- 
gang soll wahrscheinlich der silberne Stern auf dem obersten Engelskopf im Rahmen ersetzen. Aber ebenso gut hätte Herr Friedrich darüber schreiben können; Hier ist Morgen!“ Friedrich nennt den scharfsinnigen Kritiker eine „hämische Katzennatur“ und stellt klar: „Strahlend sinkt die Sonne, und im Purpur des Abendroths leuchtet der Heiland am Kreuz.“ Zugleich räumt er ein, dass „die Röthe der Luft fehlerhaft“ sei „und zweifelhaft lässt, ob es Morgen oder Abend ist.“ Es wird aber auch berichtet, Friedrich habe das Bild aus einem antinapoleonischen Impuls gemalt und König Gustav IV. von Schweden, einem erbitterten Gegner Napoleons, gewidmet. Ohne diese ältere Entstehungsgeschichte zu kennen, vermutete Hans Posse, der das Bild 1921 für die Dresdner Galerie erwarb, zum Sinngehalt: „In diesem Bild, in der Nacht vaterländischer Not entstanden, vermeint man das Symbol der Hoffnung auf einen neuen Tag zu erblicken.“ Dazu passend, sieht er „das Kreuz, einsam auf steil emporstrebender Gebirgshöhe, in der Strahlenglorie der aufgehenden Sonne“. Auch Zeitgenossen hatten mit der Tageszeit kein Problem. Der Kammerherr Carl von Voss sah das Gemälde 1822 im Schloss Tetschen und vermerkte in seinen Aufzeichnungen: „Im einfach, aber sehr vornehm eingerichteten Schlafzimmer hing über dem Bette ein schönes Bild, von Caspar David Friedrich in Dresden gemalt: ein auf einer felsigen Höhe aufgerichtetes und von den ersten Strahlen der eben aufgehenden Sonne schwach beleuchtetes Kruzifix.“

Man möchte dem spontan zustimmen, auch weil vielleicht unbewusst Dichtung in uns wirkt. 1799 erwartete Heinrich von Kleist auf der Schneekoppe den Aufgang der Sonne: „Unversiegbarer Quell, gieße die Strahlen herauf, / Helios! wälze Dein Flammenrad!“

Einen ähnlichen Strahlenkranz wie das Kreuz im Gebirge zeigt ein kleines Bild in Essen. Die meisten Kunsthistoriker werten ihn als Zeichen eines Sonnenaufgangs und titeln „Frau in der Morgensonne“. Helmut Börsch-Supan hingegen sah hier eine Abendstimmung, benannte das Bild in „Frau vor der untergehenden Sonne“ um, und stützt zudem die Interpretation auf christliches Gedankesgut: Der Weg, auf dem sie steht, wird für ihn „der Weg des Lebens“, der abrupt endet. Der Berg im Hintergrund wird als Symbol des Göttlichen verstanden.

Die aktuelle Friedrich-Forschung behauptet die Sinnoffenheit der Bilder und streitet erbittert, ob sie eine klare Botschaft ausdrücken oder nicht. Zudem wird alles mögliche Bildungsgut an Friedrich herangetragen. 1821 hatte Friedrichs Freund und Mäzen Wassili Schukowski geschrieben: „Ich bin einige Male mit ihm in der Galerie gewesen. Beim Betrachten vieler Bilder konnte er mir die Maler nicht nennen, und überhaupt ist ihm alles das, was in Lehrbüchern der Malerei enthalten ist, wenig bekannt. Dafür

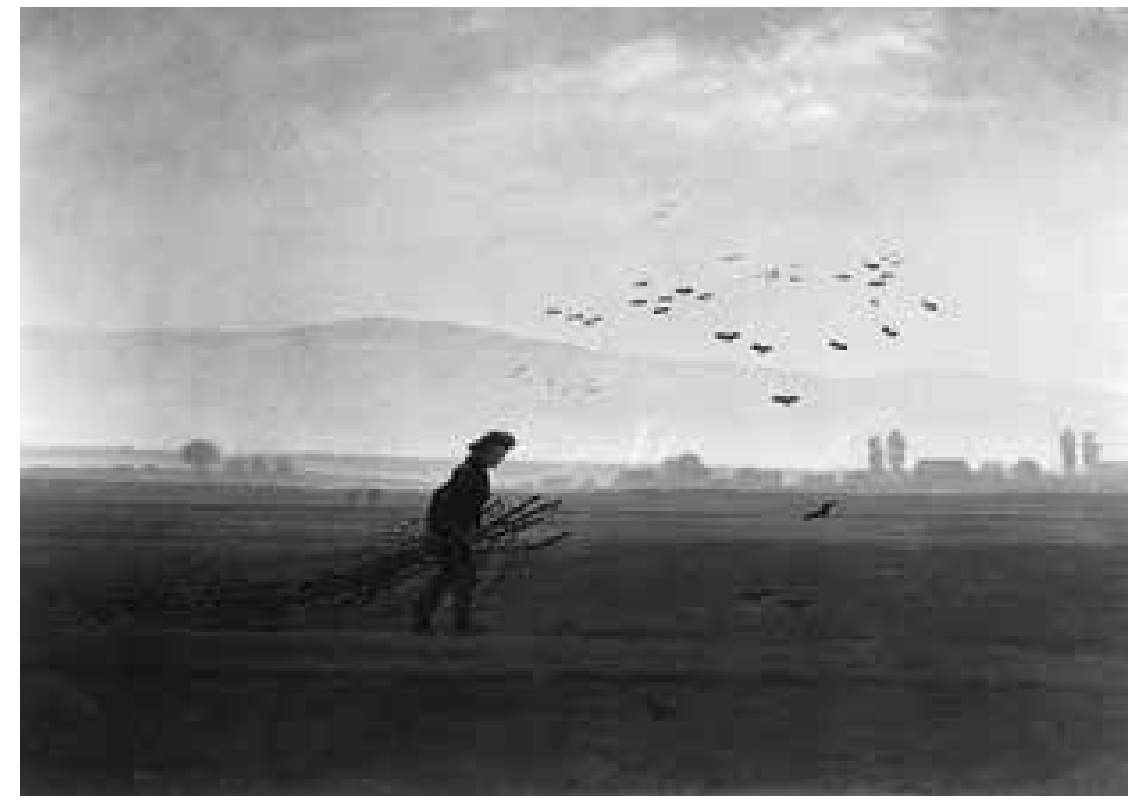

fand er in vielen Bildern Schönheiten oder Mängel, die nur der bemerkt, der in das Lehrbuch der Natur geschaut hat.“

Zweifellos macht Friedrich Denkangebote, die in Landschaftsbildern vor Augen gestellt werden. Man sollte sie aber von dem aus, was man sieht, und nicht, was man sehen möchte, beschreiben. Und nicht immer muss ein Bild von Friedrich ein durchgängiges Gedankenprogramm illustrieren. Warum soll es im einzelnen Bild nicht auch Widersprüche oder unklare Details geben? Das Schwebende, nicht genau Benennbare macht doch nicht zuletzt ihren Zauber aus. Ludwig Tiecks William Lovell klagt darüber, dass die Aufklärung allen Zauber dreist „enträtselt" habe und dass die geheimnisvolle Dämmerung einem künstlichen Tageslicht gewichen sei. „Ich hasse die Menschen, die mit ihrer kleinen nachgemachten Sonne in jede trauliche Dämmerung hineinleuchten und die lieblichen Schattenphantome verjagen, die so sicher unter der gewölbten Laube wohnten. In unserem Zeitalter ist eine Art von Tag geworden, aber die romantische Nacht- und Morgenbeleuchtung war schöner, als dieses graue Licht des wolkigen Himmels." Madame de Stael schreibt in ihrem Buch „Über Deutschland“: „Kaum haben sie [die Deutschen] einen Eindruck empfangen, so schöpfen sie schon eine Menge Ideen daraus. Sie rühmen das Geheimnisvolle sehr - aber nur, um es zu enträtseln, und man kann in Deutschland nicht die geringste Eigentümlichkeit zeigen, ohne dass es jeder auf der Stelle auseinandersetzt, woher diese Eigentümlichkeit eigentlich kommt. Das ist ein großer Nachteil - besonders für die Künste, in denen alles Empfindung ist. Sie werden eher analysiert als empfunden, und nachher predigt man vergeblich darüber, dass auf die Analyse verzichtet werden müsse.“
Caspar David Friedrich: Herbstabend mit Reisigsammler, um 1824, Öl auf Leinwand, 22 x $30,5 \mathrm{~cm}$, vormals Besitz des Prinzen Wilhelm von Preußen, $1931 \mathrm{im}$ Münchener Glaspalast verbrannt

\section{Autor}

Herrmann Zschoche Storkow 